\title{
Access Control Schema for Smart Locks using a Wifi Bridge
}

An exploration of a smart lock access control system based around the SimSim retrofitting smart lock.

\author{
Andrew Zhang \\ Icarus Systems LLC \\ andrewzhang100@gmail.com
}

\begin{abstract}
This paper presents an access control schema using a WIFI bridge for smart locks and explores its implementation in one such pair of devices. The schema outlines the interaction between a smart lock (SimSim), accompanying WIFI bridge (Freedom), a cloud server, and a group of users operating smartphones. The schema leverages AES256 symmetric encryption, Bluetooth Low Energy, and HTTPS communications to provide enhanced access control for short-term landlords participating in the sharing economy. These additional features include facilitating a larger quantity of users, 24/7 permissions management, $24 / 7$ monitoring, all without requiring physical proximity to the app.
\end{abstract}

\section{CCS CONCEPTS}

- Computer systems organization; - Embedded and cyber physical systems; $\bullet$ Embedded Systems;

\section{KEYWORDS}

Smart Locks, Robotic Actuators, Robotic Manipulators, Mechatronics, Internet of Things, Cloud

\section{ACM Reference Format:}

Andrew Zhang and Raghavendra V. P. P. Kandubai. 2020. Access Control Schema for Smart Locks using a Wifi Bridge: An exploration of a smart lock access control system based around the SimSim retrofitting smart lock. In 2020 6th International Conference on Robotics and Artificial Intelligence (ICRAI 2020), November 20-22, 2020, Singapore, Singapore. ACM, New York, NY, USA, 5 pages. https://doi.org/10.1145/3449301.3449331

\section{INTRODUCTION}

The improvement of communications technology and low-cost computation and electronics has driven the Internet of Things. In this phenomenon, many personal devices such as lighting, fridges, HVAC, and home security are being automated. Smart locks facilitate access control by allowing a user to lock and unlock their front door using either a smartphone or over the internet. [1] [2] In addition to removing the need for a physical key, they also enable additional features such as making it easier for disabled people to unlock doors, being able to give and revoke access to other people, alert owners when a door is accessed, or to unlock a door remotely [3] [4].

This work is 4.0 License.

ICRAI 2020, November 20-22, 2020, Singapore, Singapore 2020. ACM ISBN 978-1-4503-8859-7/20/11.

https://doi.org/10.1145/3449301.3449331

\author{
Raghavendra V. P. P. Kandubai \\ Project Olympus, Carnegie Mellon University \\ raghavev@tepper.cmu.edu
}

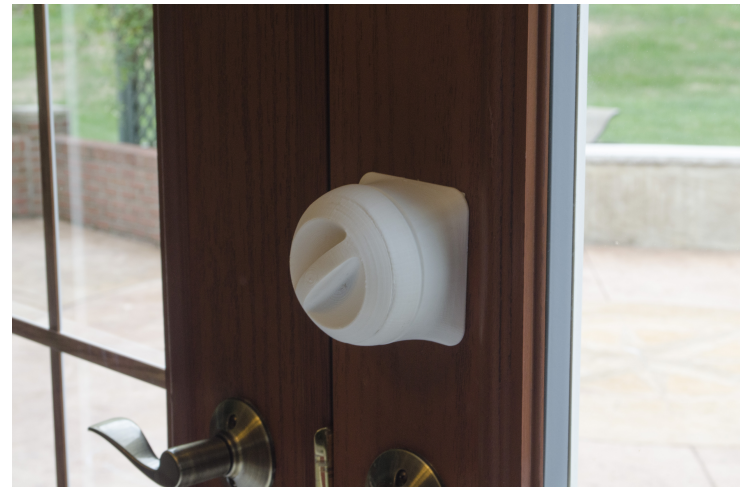

Figure 1: The retrofitting smart devices install on top of existing deadbolts.

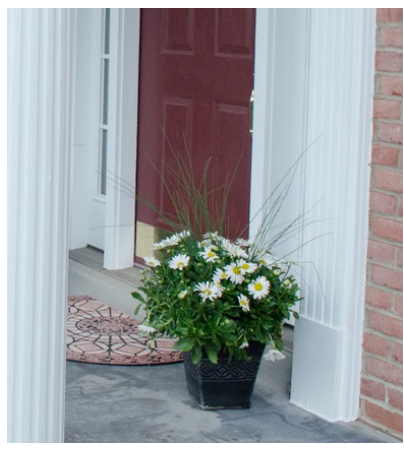

Figure 2: Landlords in the sharing economy often resort to risky security measures, such as hiding the key underneath a rug or a potted plant. Alternatively, they keep the key or leave it with nearby friends, but this forces the often-tired tenant to make a detour to find the key.

Meanwhile, there has been a parallel expansion of the sharing economy, in which ordinary people rent their belongings and homes to others. [5] These people do not have access to the resources of traditional hotels they often manage individual properties that are geographically separated. [6] As such, they do not benefit from the same economies of scale. Often, they manage their properties by hiding physical keys, which are often difficult to find, insecure, and may be copied by nefarious tenants.

These two emerging markets introduce the need for a device that can connect regular deadbolt systems to the internet and enable sophisticated access control by leveraging modern communications technology and infrastructure. [7] 


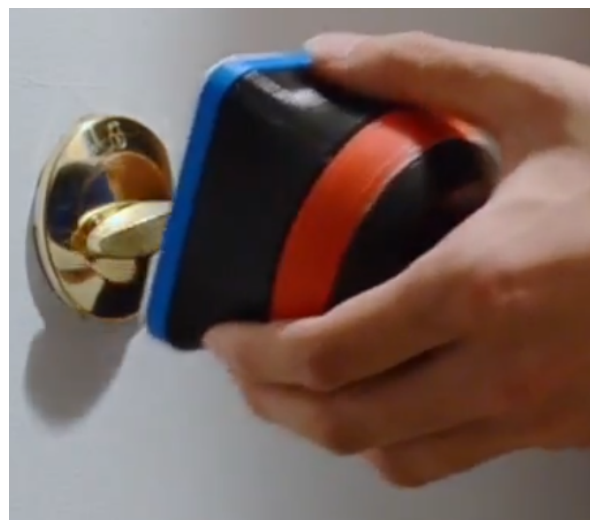

Figure 3: Retro-fitting smart device installs on top of an existing deadbolt.

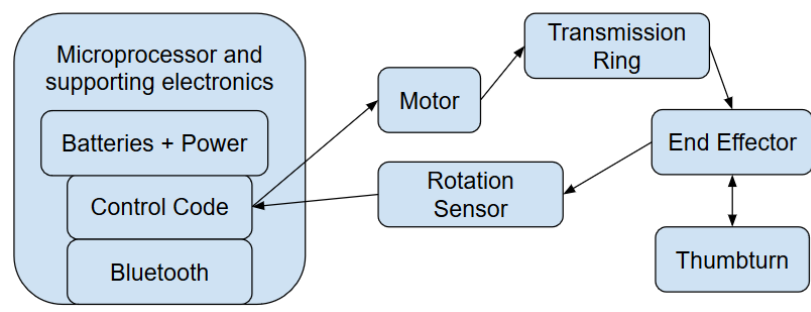

Figure 4: Overall architecture of a retro-fitting smart-device.

\section{OVERALL ARCHITECTURE}

The hardware used for this approach was SimSim, a smart device that can be retrofitted on top of an existing deadbolt and operates a deadbolt thumbturn as if it were a robotic hand (figure 3). In this way, it can leverage the existing deadbolt's security. Note that it is not a true "smart-lock" (as the term "lock" would refer to the underlying deadbolt mechanism) but rather a "robotic lock actuator". However, since it takes the place of a typical smart lock in the context of access control, it will be referred to as such during this paper.

More relevant to its application in sharing, is that it is much simpler to install and cheap to purchase. This is important since the owner of the property being shared is not a large entity such as a hotel, and as such has access to far fewer resources.

Like most smart locks, SimSim is equipped with a central microprocessor to control the hardware, a motor to manipulate the lock, and a rotation sensor (more specifically, an accelerometer) to measure the position of the lock, and a wireless radio. (Figure 4)

In addition to these features, SimSim has a suite of features that facilitate its retrofitting feature [8]:

1. an adjustable end-effector ("gripper") that accounts for misalignment in the axis of rotation between the smart device and the deadbolt thumbturn

2. a rotating body design that can simplify the structure and reduce cost while improving reliability, by rotating the entire

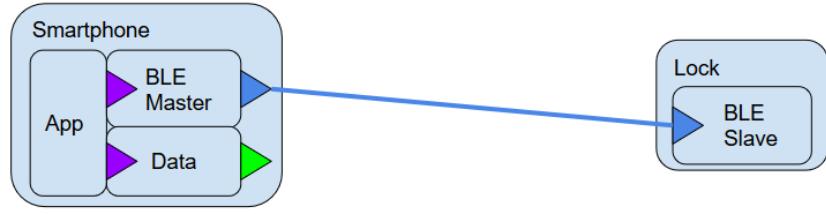

Figure 5: Smartphone direct connection. Triangles represent compatible connections (blue $=$ BLUE, purple $=$ software, green $=$ HTTPS)

electronics assembly within a passive shell, instead of only rotating the gripper

3. a retreating transmission ring that allows manipulation of the lock manually (both internally through the body of the smart lock, and externally using a physical key) while also being able to detect this manual operation.

\section{WIFI CONNECTIVITY}

The wireless radio used on SimSim is a Bluetooth Low Energy (BLE) radio, chosen for its low power consumption and integration into modern smartphones (figure 5). However, this BLE radio is unable to communicate with the internet, which introduces the need for a WIFI bridge named Freedom (figure 6). The WIFI bridge replaces the smartphone and extends the maximum range from roughly $30 \mathrm{~m}$ to anywhere with an internet connection (figure 7).

One design tradeoff of the design of the Freedom lock is that BLE radios are only able to connect to one other device at a time. Part of this restriction is that the protocol uses a master/slave architecture.

For most applications (explored through the implementation of an early prototype of Freedom) there is only one BLE radio and one WIFI radio. While the WIFI bridge is active, the user would be unable to connect to the smart lock through BLE. This requires the user to have a cellular connection. However, this is hidden from the user through the app interface, so long as the phone can connect the lock somehow, and thus the user experiences a seamless user experience. This approach also allows the implementation of a bridge by repurposing an off-the-shelf smartphone, greatly reducing development costs.

However, in the context of access control to vacation homes, many of the users may be in a foreign country or in a remote location without a data connection. As such, they would still need to be able to connect to the lock through BLE. To fulfill the requirement, Freedom was given two BLE radios, one to connect to the physical lock (as a master), and another to connect to the smartphone (as a slave). (Figure 8)

\section{SMARTLOCK AUTHENTICATION}

Although BLE comes with its own encryption this protocol relies mostly on its own layer of AES256 encryption, both to increase security, and to facilitate additional features. AES256 is a symmetric encryption method, that requires a shared 256-bit key to both encrypt and decrypt a message. It has yet to be cracked as of 2020) [9]. 


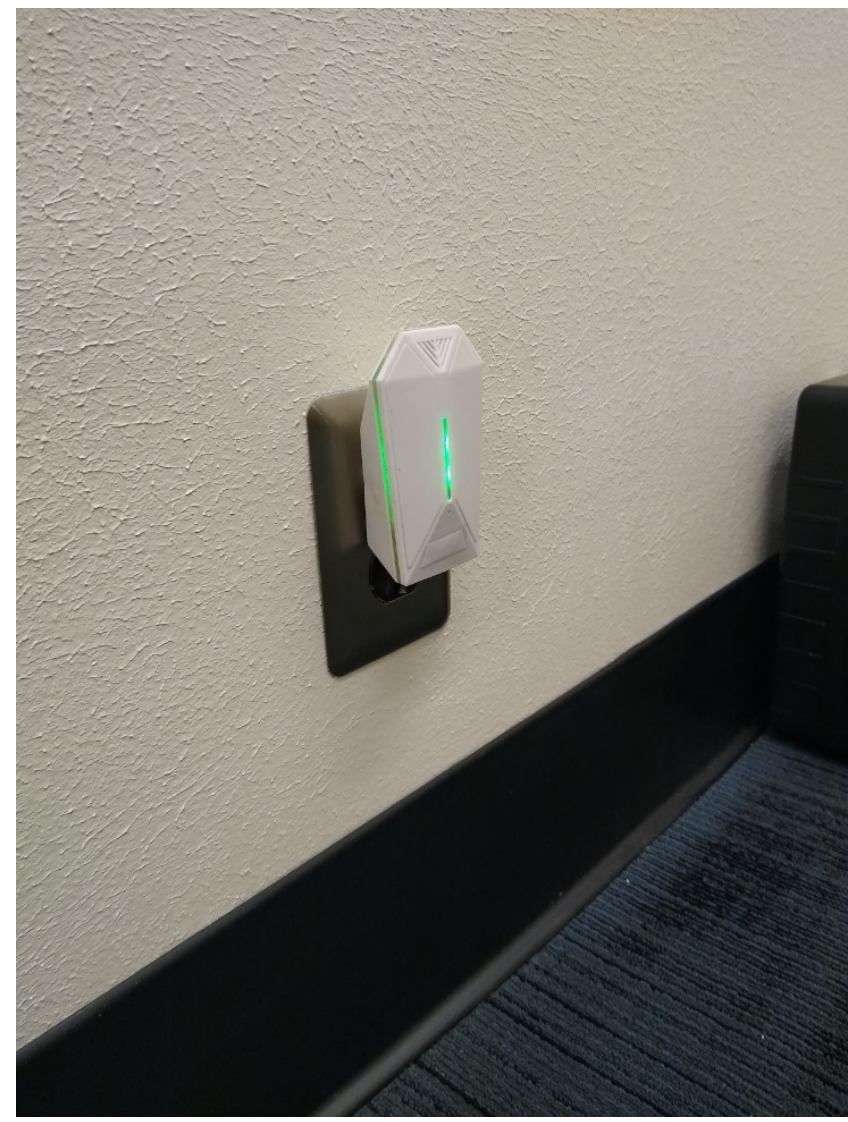

Figure 6: Freedom is plugged into a power outlet near the lock and provides WIFI connectivity without adding the relatively power-draining hardware on the lock itself. While BLE has a nominal maximum range of $100 \mathrm{~m}$, in practice, the signal begins to deteriorate around $30 \mathrm{~m}$, and an even shorter distance if the signal needs to travel through a door.

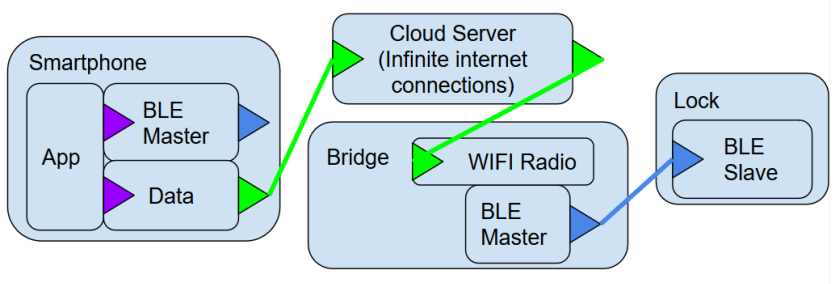

Figure 7: Smartphone connection over the bridge, with one BLE Master Radio. (blue $=$ BLE, purple $=$ software, green = HTTPS).

\subsection{Regular Use}

1 A "user" (someone using the smartphone app) will send the lock a message, with the ID and encrypted using its AES256 key. (Figure 9)

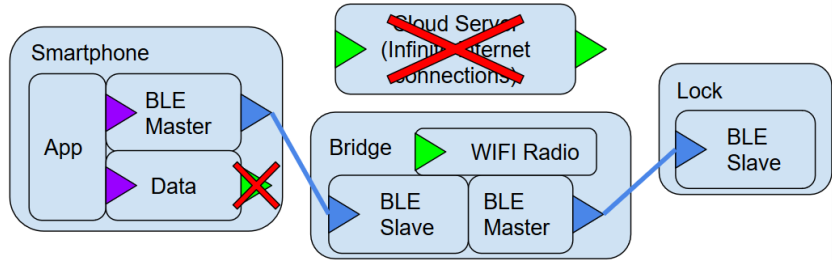

Figure 8: Smartphone connection to smart lock with the bridge, but the internet is unavailable, and/or the smartphone is unable to connect to the internet. Not depicted here is the microprocessor located on the bridge that coordinates the three radios and processes data that passes through it. (blue $=$ BLE, purple $=$ software, green $=$ HTTPS $)$.

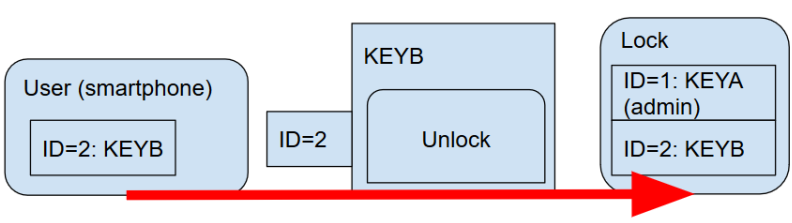

Figure 9: Both the user and the lock have KEYB under ID=2. The user sends an unlock command, encrypted with KEYB, together with $I D=2$. KEYB is not sent in this command. The lock will look up ID $=2$ to find KEYB and use it to unencrypt the message, and then unlock.

2 The lock will then look up the ID in its database, and unencrypt the message using the key associated with that ID.

3 If the key can successfully decrypt the message, the lock will then follow the commands in the message.

4 If required, the lock may respond with a status message, encrypted with that key. The user will then unencrypt that message with the key that they have.

\subsection{Admin Users}

The smart lock also has a series of "administrator" (admin) keys. These owners of these admin keys further have the permission to add new keys and IDs to the lock, delete them, and set admin status, in the form of encrypted commands using their key. (Figure 10) The admin can even set a user to expire after a certain time, or disable special features such as proximity unlocking (the lock unlocks automatically if the user approaches it) or long-distance access, which is particularly useful for rental properties.

When the lock is first initiated, it will be in a special "initialized" state. If a user connects to it in this state (over an "unencrypted" connection), it can send it a key that the lock will accept as an admin key. In this way, the first user to connect to the lock will be an admin user.

\subsection{User Interface}

Note that the above protocols are hidden from the end-user. Instead, the end-user uses an app, which stores keys locally while also 


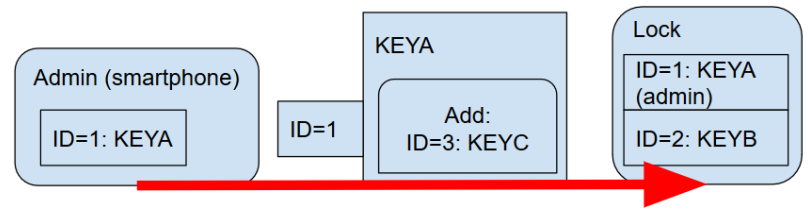

Figure 10: Admin $(I D=1)$ commands the lock to add " $I D=3$ : KEYC" to its list of authenticated users, over its secure communications channel encrypted by KEYA.

(optionally) backing them up to a trusted cloud server. In this way, the user can access their key using a traditional username and password, instead of being exposed to the AES256 keys directly.

\subsection{Hardware Limitations}

Each user occupies 40B, which consists of a 32B key, and some other metadata. The lock, which is powered by an atmega328p, has 1024B of non-volatile EEPROM and can store up to 24 users. The remaining 64B of EEPROM is allocated to other non-volatile parameters such as the angles at which the deadbolt thumbturn is closed or opened.

\section{BRIDGE AND SERVER INTEGRATION}

Since the WIFI bridge has a master BLE radio much like a smartphone, it can show up to the manipulator as simply another admin "user". The bridge can pair and connect to the manipulator the same way as any smartphone (the setup is done through the app, indirectly through the internet). Meanwhile, it automatically connects to a cloud server.

The implementation of the bridge in this way means that there are no additional modifications over operation without the bridge, either in hardware or in software to the manipulator itself, simplifying the development of the manipulator. Further, it allows the lock to be controlled by an infinite number of additional users, while only consuming one "position" on the limited memory of the manipulator itself.

The implementation of server access on the phone also shows up as an additional lock, reducing the additional development on the app to add this feature.

If the bridge is connected directly using a phone (as in figure 8), it simply allows pass-through communication, which causes it to appear to the phone as the lock, and to the lock as the phone connected to it. This allows the user to use the bridge to control the lock even if the internet is unavailable. Alternatively, if the bridge loses power, the BLE radio on the lock itself frees up and can be connected directly with the phone.

\section{CLOUD-LEVEL ACCESS CONTROL}

All bridges and apps "call home" to the cloud server (this time using the SSL encryption inherent to HTTPS communications), so the cloud server can implement the following features:

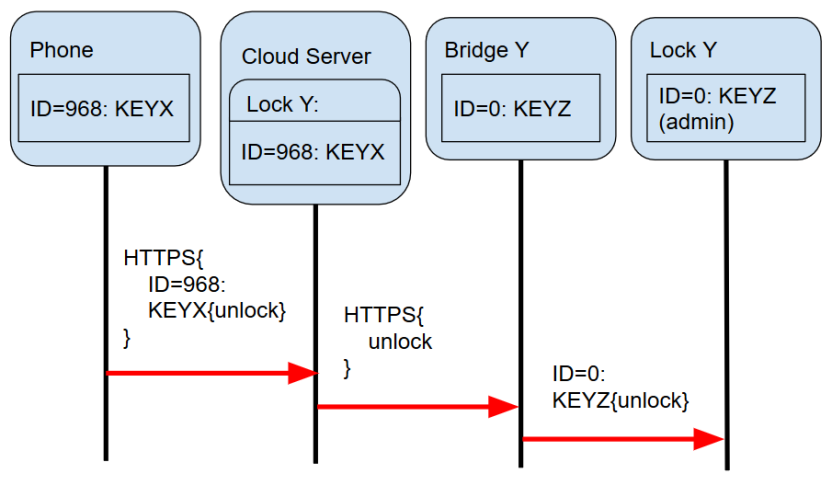

Figure 11: The cloud server uses the bridge to operate the lock. Although the lock can only store 24 users, the cloud server can store an infinite number of users and is also trusted by the bridge. Although the lock is unable to see who sent the command, the cloud server can and will log this information.

\subsection{Indefinite Quantity of Users}

Since the cloud server is not restricted by the same hardware limitations as the lock, it can store an indefinite number of keys and key IDs. If a user attempts to control a lock, it sends its key to the cloud server, which, should the key be accepted, commands the bridge to use its key to control the lock.

The downside of this implementation is that this indefinite number of users is unavailable to the bridge if internet is unavailable. One improvement is to move the "indefinitely large key database" to the bridge, allowing access to the greater number of users even when the Internet is unavailable (figure 12). While the bridge has hardware limitations unlike the cloud server, these limitations are not as extreme as the lock itself, given the reduced size constraints, access to a power outlet, and reduced development cost from the relative simplicity of the bridge. As such, it could house a small flash chip for storing user keys and other data. However, this approach was not implemented in the overall system to speed up the bridge's hardware development.

\subsection{4/7 Access Control}

Since the lock is now connected to the internet through the bridge, (as in figure 7) it can be controlled even if the user is not physically near the lock (for a direction connection as shown in figure 5). For example, if someone without access requests someone with access to open the lock, the person with access can do so remotely. (This is a permission that can be set). Alternatively, an admin can remove access from another user without needing to go to the property.

\section{$6.324 / 7$ Monitoring}

The lock will also be constantly connected to the bridge, and so will be constantly communicating its state to the bridge. When the bridge forwards these messages to the cloud server, the cloud server can record these messages, so that the admins can check or be notified whenever the lock has been operated, a) manually (figure 13), b) by a user over the BLE connection on the bridge 


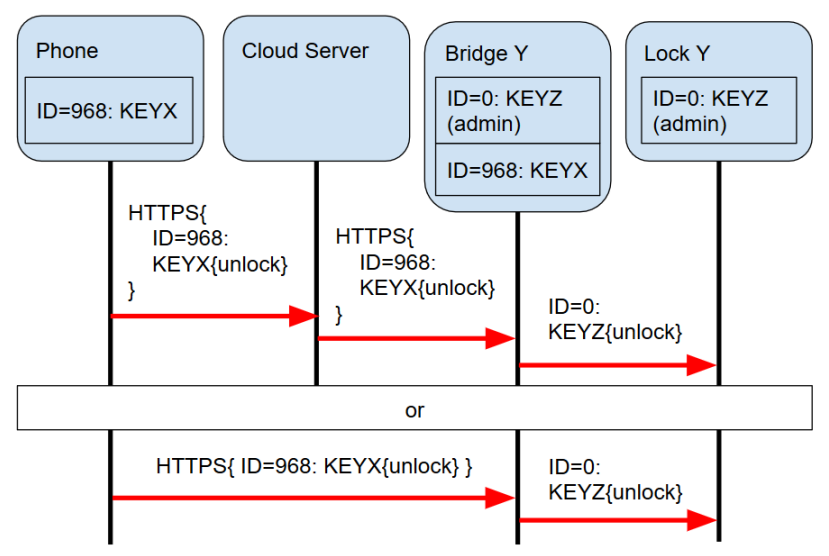

Figure 12: Storing keys on the bridge instead of the cloud server would allow access even if the cloud server is unavailable. This is vital in case the user does not have an internet connection, and also their user data is not on the lock.
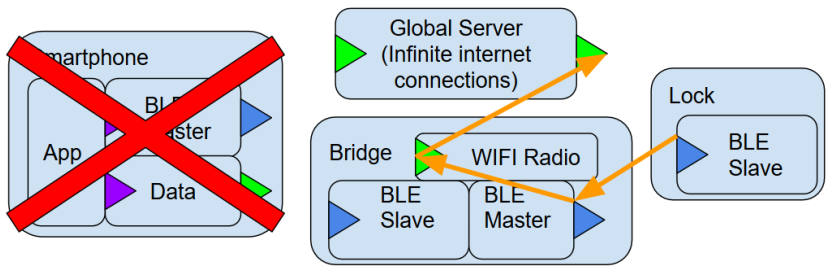

Figure 13: Manual operation of the lock is still reported to the cloud server if the lock is connected to a bridge. If the bridge and/or lock are unavailable, the cloud server would $\log$ this time as "disconnected".

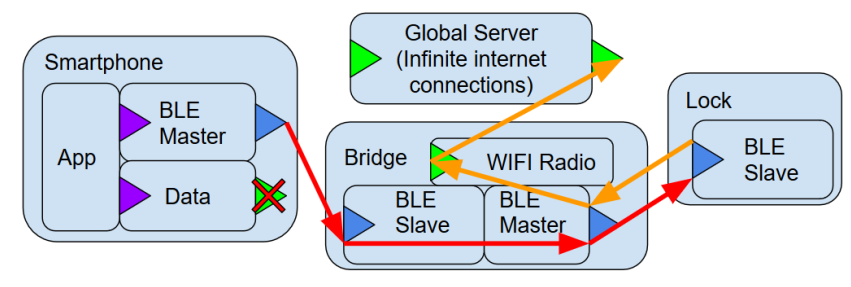

Figure 14: A user without an internet connection controls the lock (red), and the lock can report this usage to both the user and the cloud server (orange). Since the lock reports this usage to the cloud server, the owner of the lock will be able to see this activity.

(figure 14), c) by a user over the cloud server. (note that due to the hardware limitations of the lock, it cannot tell whether the lock was operated from the inside or using a key from the outside).

\section{CONCLUSION}

This paper explains the implementation of enhanced access control for property sharing. It enables multiple levels of permissions and secure control of the lock. With the use of a bridge, it also allows $24 / 7$ control and monitoring of the lock regardless of proximity, and more flexible permissions granting and revocation, in a way that does not complicate the existing hardware and firmware of the lock.

However, while promising, this schema is untested on a large scale, where its features become more applicable.

For continued development, the extended storage of keys should be moved to the bridge instead of the cloud server to enable greater accessibility, as noted in Section 6.1.

Further, the cloud server in its current implementation has too much access, and most individuals would be hesitant to trust a third party to unlock their properties. As such, the ability to control a lock must be held under an additional layer of authentication so that the cloud server is only able to forward pre-encrypted commands, and the logs that record the log monitoring the locks must not be accessible without a key that only the user can provide. The storage of user keys and other data on the bridge instead of the cloud server would also support this endeavor.

\section{ACKNOWLEDGMENTS}

Thanks to Kit Needham and Project Olympus for allowing us to use their space, and for providing valuable and priceless consultation.

\section{REFERENCES}

[1] Abdallah Kassem, Sami El Murr, Georges Jamous, Elie Saad, Marybelle Geagea, 2016. A smart lock system using Wi-Fi security. 222-225. 10.1109/ACTEA.2016.7560143.

[2] Adarsh V Patil, Sreevarsha Prakash, Akshay S, Mahadevaswamy, Chandanb Patgar, Sharath Kumar A J, 2018, Android Based Smart Door Locking System, International fournal of Engineering Research \& Technology (IfERT) NCESC - 2018 (Volume 6 Issue 13),

[3] Muhammad Sabirin Hadis; Elyas Palantei; Amil Ahmad Ilham; Akbar Hendra, Mar. 2018. Design of smart lock system for doors with special features using bluetooth technology, IEEE International Conference on Information and Communications Technology (ICOIACT), Yogyakarta, 2018, pp. 396-400, DOI= 10.1109/ICOIACT.2018.8350767.

[4] N.H. Ismail; Zarina Tukiran; N.N. Shamsuddin, 2014, Android-based home door locks application via Bluetooth for disabled people, IEEE International Conference on Control System, Computing and Engineering (ICCSCE 2014), Batu Ferringhi, 2014, pp. 227-231, DOI= 10.1109/ICCSCE.2014.7072720.

[5] Juho Hamari, Mimmi Sjöklint, Antti Ukkonen, 2016, The Sharing Economy: Why People Participate in Collaborative Consumption, fournal of the Association for Information Science and Technology. 67. 2047-2059. 10.1002/asi.23552.

[6] Shrirang Mare, Franziska Roesner, Tadayoshi Kohno, 2020, Smart Devices in Airbnb: Considering Privacy and Security for both Guests and Hosts, Sciendo, Proceedings on Privacy Enhancing Technologies, 2020 (2). 436-458. DOI $=10.2478$ /popets-2020-0035.

[7] SimSim, Oct 2019, andrewzhang.ca/mechatronics/x-htm

[8] Andrew Zhang, Raghavendra Kandubai, Stowe Hammarberg, 2020, A Functional Retro-Fitting Robotic Smart Lock Manipulator, unpublished.

[9] Bi Irie guy-cedric, Toa. (2018). A Comparative Study on AES 128 BIT AND AES 256 BIT. INTERNATIONAL JOURNAL OF COMPUTER SCIENCES AND ENGINEERING. volume 6. 30-33. 10.26438/ijsrcse/v6i4.3033. 